\title{
SELETIVIDADE DE AGROTÓXICOS RECOMENDADOS NA PRODUÇÃo INTEGRADA DA MAÇÃ A Trichogramma pretiosum RILEY, 1879 (HYM.: TRICHOGRAMMATIDAE) EM CONDIÇÕES DE LABORATÓRIO ${ }^{1}$
}

\author{
CRISTIANE GINDRI MANZONI²*, ANDERSON DIONEI GRÜTZMACHER ${ }^{3}$, FABRIZIO PINHEIRO GIOLO ${ }^{2}$, \\ CRISLAINE ALVES BARCELLOS DE LIMA ${ }^{4}$, SANDRO DANIEL NÖRNBERG ${ }^{5}$, WAGNER DA ROZA HÄRTER ${ }^{6}$, \\ CRISTIANE MÜLLER ${ }^{7}$
}

RESUMO - A seletividade de oito agrotóxicos recomendados na Produção Integrada da Maçã (PIM) foi avaliada sobre Trichogramma pretiosum Riley em laboratório, utilizando-se da metodologia padronizada pela International Organization for Biological and Integrated Control of Noxious Animals and Plants (IOBC). Os produtos (\% da formulação comercial na calda) Captan 500 PM $(0,240)$ e Delan $(0,125)$ foram inócuos; Promalin $(9,000)$ foi levemente nocivo; Assist $(4,000)$ e Dormex $(1,200)$ foram moderadamente nocivos; Vertimec 18 CE $(0,100)$, Malathion 1000 CE $(0,100)$ e Supracid 400 CE $(0,100)$ foram nocivos a adultos de $T$. pretiosum no teste de toxicidade em laboratório. Os agrotóxicos classificados como moderadamente nocivos e nocivos requerem testes subsequientes de persistência biológica do produto em casa de vegetação e de campo.

Termos para indexação: manejo integrado de pragas, IOBC, agrotóxicos, macieira.

\section{SELECTIVITY OF PESTICIDES USED IN INTREGATED PRODUCTION OF APPLE ON Trichogramma pretiosum RILEY, 1879 (HYM.: TRICHOGRAMMATIDAE) IN LABORATORY CONDITIONS}

\begin{abstract}
The side effects of eight pesticides recommended in the Integrated Production of Apple (IPA) was evaluated on Trichogramma pretiosum Riley in laboratory, using the International Organization for Biological and Integrated Control of Noxious Animals and Plants (IOBC) standard methodology. The pesticides (\% commercial formulation in spray water) Captan 500 PM (0.240) and Delan (0.125) were harmless; Promalin (9.000) was slightly harmful; Assist (4.000) and Dormex (1.200) were moderately harmful; Vertimec 18 CE (0.100), Malathion 1000 CE (0.100) and Supracid 400 CE (0.100) were harmful to parasitoids in toxicity test on egg parasitoid T. pretiosum. The pesticides classified as moderately harmful and harmful, request subsequent tests of biological persistence of the product in greenhouse and on field.
\end{abstract}

Index terms: integrated pest management, IOBC, pesticides, apple tree.

\section{INTRODUÇÃO}

Os Estados de Santa Catarina e Rio Grande do Sul são os principais produtores de maçã do Brasil, respondendo por cerca de 96\% da produção nacional, que na safra de 2004 foi de aproximadamente 973 mil toneladas, com rendimento médio de 29,63 toneladas.ha ${ }^{-1}$ (IBGE, 2005). No entanto, a produção de maçã é a cada safra comprometida pela ação de insetos-praga com destaque para Bonagota cranaodes (Meyrick, 1937) (Lepidoptera: Tortricidae) e Grapholita molesta (Busck, 1916) (Lepidoptera: Tortricidae) (Kovaleski $\&$ Ribeiro, 2002).

O controle destes insetos-praga geralmente requer inúmeras aplicações de inseticidas, principalmente de organofosforados, que apresentam sérias restrições de uso, além de afetar insetos benéficos presentes nos pomares. Além disso, a cultura destaca-se pelo grande número de aplicações de outros agrotóxicos, principalmente fungicidas e reguladores de crescimento de plantas, que também podem afetar agentes de controle biológico das referidas pragas. Dentre os inimigos naturais, merecem destaque os parasitóides de ovos da família Trichogrammatidae, em especial do gênero Trichogramma, que apresentam distribuição mundial, ocorrendo associados a diversas espécies de insetos-praga em diferentes cultivos. Trichogramma pretiosum Riley, 1879 é a espécie mais amplamente distribuída na América do Sul, além de ser a mais polífaga, tendo sido associada a 26 espécies (Pinto, 1997), incluindo G. molesta (Pinto et al., 2002) e $B$. cranaodes (Monteiro et al., 2004), em pomares comerciais de macieira.

Na Produção Integrada da Maçã (PIM), são preconizadas ações que visam a conservar os inimigos naturais, entre as quais o uso de agrotóxicos seletivos, que controlem eficientemente as pragas, sem prejudicar as populações de inimigos naturais. Segundo Kovaleski et al. (2000), é provável que, após algumas safras com menor volume de aplicações de agrotóxicos, as áreas de PIM venham a exibir populações maiores de organismos benéficos, favorecendo o controle biológico.

No Brasil, estudos visando a conhecer o efeito de agrotóxicos sobre organismos benéficos da cultura da macieira foram realizados somente com predadores, como, por exemplo, com o ácaro Neoseiulus californicus (McGregor, 1954) (Acari: Phytoseiidae) (Monteiro, 2001) e com o inseto Chrysoperla externa (Hagen, 1861) (Neuroptera: Chrysopidae) (Ferreira et al., 2005). No entanto, até o presente momento, nenhum trabalho foi realizado para avaliar o efeito de agrotóxicos recomendados na PIM sobre parasitóides de ovos do gênero Trichogramma. Devido a sua relevância como importante agente de controle biológico de insetos-praga, este gênero foi selecionado pelo grupo de trabalho da "International Organization for Biological and Integrated Control of Noxious Animals and Plants (IOBC), West Palaearctic Regional Section (WPRS)", como um dos padrões a serem utilizados em testes de seletividade obrigatórios para registro de novos agrotóxicos na União Européia (Hassan et al., 2000).

Considerando o potencial de utilização deste parasitóide associado ao controle químico na PIM, realizou-se este trabalho com o objetivo de avaliar a seletividade de oito agrotóxicos recomendados na PIM a adultos de T. pretiosum, em condições de laboratório.

\section{MATERIAL E MÉTODOS}

Os experimentos foram executados de acordo com a metodologia laboratorial padrão da IOBC/WPRS (Hassan et al., 2000).

\footnotetext{
${ }^{1}$ (Trabalho 118-2005). Recebido: 29-07-2005. Aceito para publicação: 18-04-2006. Trabalho realizado com apoio financeiro do CNPq.

${ }^{2}$ Eng. Agr., Doutorando do PPGFs/Depto. de Fitossanidade/FAEM/UFPel. "e-mail: cristianemanzoni @ hotmail.com.

${ }^{3}$ Eng. Agr., Dr., Prof. Depto. de Fitossanidade/FAEM/UFPel. email: adgrutzm@ufpel.tche.br.

${ }^{4}$ Bióloga, Bolsista de Apoio técnico - NS, CNPq/FAEM/UFPel. email: clima@ ufpel.tche.br.

${ }^{5}$ Acadêmico Agronomia, BIC FAPERGS/Depto. de Fitossanidade/FAEM/UFPel. email: sandronornberg@ @erra.com.br.

${ }^{6}$ Acadêmico Agronomia, BIC CNPq/Depto. de Fitossanidade/FAEM/UFPel. email: wharter.faem@ufpel.tche.br.

${ }^{7}$ Acadêmica Agronomia, Bolsista PET/CAPES. email: crismiler@yahoo.com.br.
} 
Os parasitóides $T$. pretiosum foram provenientes de criação mantida em laboratório (temperatura $25 \pm 1^{\circ} \mathrm{C}$, umidade relativa $70 \pm 10 \%$ e fotofase de 14 horas) sobre ovos do hospedeiro alternativo Anagasta kuehniella (Zeller, 1879) (Lepidoptera: Pyralidae), criado segundo metodologia descrita por Parra (1997).

Em função da capacidade operacional do sistema instalado no laboratório, os estudos de seletividade foram divididos em dois experimentos. Os testes de toxicidade foram conduzidos em condições laboratoriais (temperatura $25 \pm 1^{\circ} \mathrm{C}$, umidade relativa $70 \pm 10 \%$ e fotofase de 14 horas), expondo-se adultos (estágio mais sensível) de $T$. pretiosum a resíduos secos de agrotóxicos, utilizando-se da máxima dosagem recomendada na PIM (Tabela 1). O inseticida Dipterex 500 foi utilizado como padrão de toxicidade em todos os experimentos, por ser reconhecidamente nocivo a parasitóides do gênero Trichogramma (Grützmacher et al., 2004), enquanto a testemunha foi constituída por água. Os produtos foram aplicados diretamente sobre placas de vidro de $2 \mathrm{~mm}(13 \times 13 \mathrm{~cm})$ através de pulverizadores manuais (580 mL) calibrados para depositar o volume de 1,75 $\pm 0,25 \mathrm{mg}$ de calda por $\mathrm{cm}^{2}$ de superfície, aferido através de balança eletrônica de precisão. Depois de tratadas, as placas permaneceram à temperatura ambiente por três horas para secagem, onde a calda aplicada formou uma película seca. Gaiolas de contato foram confeccionadas utilizando-se de duas placas tratadas, fixas a uma moldura retangular de alumínio $(13 \mathrm{~cm}$ de comprimento x $1,5 \mathrm{~cm}$ de altura $\mathrm{x} 1 \mathrm{~cm}$ de largura), através de presilhas. Em três dos lados da moldura de alumínio, havia orifícios para ventilação (diâmetro aproximado de $1 \mathrm{~cm}$ ). O quarto lado da moldura possuía dois orifícios: um $(3,5 \times 1 \mathrm{~cm})$ para introdução dos ovos do hospedeiro A. kuehniella a serem parasitados e alimento (composto por $3 \mathrm{~g}$ de gelatina, $100 \mathrm{~mL}$ de água e $200 \mathrm{~g}$ de mel) para os insetos em teste, e o outro (diâmetro de $1 \mathrm{~cm}$ ) para a introdução dos parasitóides, através da conexão dos tubos de emergência.

Cada tubo de emergência (ampola de vidro transparente com $120 \mathrm{~mm}$ de comprimento $\mathrm{x} 20 \mathrm{~mm}$ de diâmetro em uma das extremidades e $7 \mathrm{~mm}$ na outra) foi constituído por um círculo ( $1 \mathrm{~cm}$ de diâmetro) contendo $250 \pm 50$ ovos previamente parasitados, aderido a uma tira de papel $(80 \times 15 \mathrm{~mm})$ com alimento. Esses tubos contendo adultos de T. pretiosum com aproximadamente 24 horas de idade foram conectados às gaiolas de contato seis horas após a pulverização dos produtos, permitindo a entrada dos parasitóides no interior das gaiolas. Transcorridas 16 horas, os tubos foram desconectados e mantidos sob as mesmas condições do experimento por mais três dias para que ocorresse a total emergência dos adultos. Para evitar o aumento na concentração de gases tóxicos, no interior das gaiolas de exposição foi acoplado, através de mangueiras, um sistema de sucção de ar constituído por bombas de aquário com fluxo invertido.

Seis horas após a desconexão dos tubos de emergência, foram oferecidos os primeiros cartões com ovos de A. kuehniella (cada cartão contendo 3 círculos de $1 \mathrm{~cm}$ de diâmetro, com $400 \pm 50$ ovos) e alimento. Os cartões foram oferecidos para serem parasitados 24 (três cartões), 48 (dois cartões) e 96 (um cartão) horas após a pulverização, perfazendo um período aproximado de 144 horas, com disponibilidade de ovos para o parasitismo. Sete dias após a pulverização, as gaiolas foram desmontadas. Os cartões oferecidos ao parasitismo foram armazenados nas mesmas condições do teste por mais três dias, período suficiente para que houvesse o escurecimento de todos os ovos parasitados.

Para determinar o número de fêmeas em cada gaiola, foram contados os ovos parasitados em cada círculo utilizado na confecção dos tubos de emergência. Este valor foi multiplicado pelo número de parasitóides por ovo da população do inseto. $\mathrm{O}$ valor obtido foi subtraído do número de adultos que permaneceram no interior do tubo de emergência e multiplicado pela razão sexual da população. Os parâmetros número de parasitóides por ovo e razão sexual foram obtidos mediante avaliação de 4 círculos de $1 \mathrm{~cm}$ de diâmetro contendo ovos parasitados, sendo estes círculos retirados do mesmo cartão utilizado na confecção dos tubos de emergência.

O número médio de ovos parasitados por fêmea em cada tratamento foi utilizado para calcular a capacidade de parasitismo. A redução na capacidade de parasitismo para cada agrotóxico foi determinada através da comparação com a testemunha (água destilada) e calculada por meio da fórmula $\mathrm{RP}=\left[1-(P / p)^{*} 100\right]$, onde RP é a porcentagem de redução no parasitismo, $P$ é o valor do parasitismo médio para cada produto e $p$ o parasitismo médio observado para o tratamento-testemunha. Com base nestas porcentagens de reduções no parasitismo, os produtos testados foram classificados, segundo IOBC/WPRS, em: 1) inócuo (<30\%); 2) levemente nocivo (30-79\%); 3 ) moderadamente nocivo ( $80-99 \%$ ), e 4 ) nocivo ( $>99 \%$ ). Cada gaiola de contato foi considerada uma unidade experimental. Os experimentos foram inteiramente casualizados e delineados com quatro repetições por tratamento. Os dados obtidos, referentes ao número de ovos parasitados por fêmea, foram submetidos à análise de variância, e as médias dos tratamentos, comparadas pelo teste de Tukey $(\mathrm{P}<0,05)$.

\section{RESULTADOS E DISCUSSÃO}

O número médio de fêmeas de $T$. pretiosum no interior das gaiolas de exposição ficou entre 105,19 e 213,51 no Experimento I e entre 98,48 e 137,96 no Experimento II (Tabela 2). Estes resultados estão de acordo com a metodologia da IOBC/WPRS, que recomenda um número entre 50 e 400 parasitóides por gaiola de contato, para testes de seletividade em laboratório, com Trichogramma cacoeciae Marchal, 1927 (Hymenoptera: Thichogrammatidae) (Hassan, 1998a; Hassan et al., 2000) para uma adequada mensuração dos resultados, que são expressos em número de ovos parasitados por fêmea. $\mathrm{O}$ número médio de ovos parasitados por fêmea de T. pretiosum variou significativamente entre os produtos testados (Tabela 2). $\mathrm{Na}$

TABELA 1 - Agrotóxicos avaliados nos testes de seletividade a adultos de Trichogramma pretiosum, utilizando a dosagem máxima do produto formulado recomendado pela Comissão Técnica da Produção Integrada da Maçã (CTPIM).

\begin{tabular}{|c|c|c|c|c|c|c|}
\hline Ingrediente ativo & Formulação comercial & Classe $^{\mathrm{T}}$ & Grupo químico & $\mathrm{DC}^{2}$ & C.i.a. $^{3}$ & C.f.c. ${ }^{4}$ \\
\hline Abamectina & Vertimec $18 \mathrm{CE}$ & $\mathrm{A} / \mathrm{I}$ & Avermectinas & 100 & 0,002 & 0,100 \\
\hline Óleo mineral & Assist & $\mathrm{A} / \mathrm{I}$ & Hidrocarbonetos & 4000 & 3,024 & 4,000 \\
\hline Captana & Captan 500 PM & $\mathrm{F}$ & Dicarboximida & 240 & 0,113 & 0,240 \\
\hline Ditianona & Delan & $\mathrm{F}$ & Quinona & 125 & 0,094 & 0,125 \\
\hline Malationa & Malathion $1000 \mathrm{CE}$ & I & Organofosforado & 100 & 0,100 & 0,100 \\
\hline Metidationa & Supracid $400 \mathrm{CE}$ & I & Organofosforado & 100 & 0,040 & 0,100 \\
\hline Triclorfom & Dipterex 500 & I & Organofosforado & 300 & 0,150 & 0,300 \\
\hline Cianamida & Dormex & $\mathrm{RCP}$ & Carbimida & 1200 & 0,624 & 1,200 \\
\hline Benziladenina + Ácido Giberélico & Promalin & $\mathrm{RCP}$ & Citocinina + Giberelina & 9000 & $0,1692+0,1692$ & 9,000 \\
\hline
\end{tabular}

${ }^{1} \mathrm{~A}=$ acaricida; $\mathrm{I}=$ inseticida; $\mathrm{F}$ = fungicida; $\mathrm{RCP}$ = regulador de crescimento de plantas;

${ }^{2} \mathrm{DC}=$ Dosagem de campo $\left(\mathrm{g}\right.$ ou $\mathrm{mL}$ do produto comercial. $\left.100 \mathrm{~L}^{-1}\right)$;

${ }^{3}$ C.i.a. $=$ Concentração $(\%)$ testada do ingrediente ativo na calda;

${ }^{4}$ C.f.c. $=$ Concentração $(\%)$ testada da formulação comercial na calda. 
TABELA 2 - Efeito de agrotóxicos recomendados pela Comissão Técnica da Produção Integrada da Maçã (CTPIM) sobre o número médio ( \pm EP) de ovos parasitados por fêmea, redução (\%) na capacidade de parasitismo de Trichogramma pretiosum e classificação de toxicidade destes produtos. Temperatura $25 \pm 1^{\circ} \mathrm{C}$; UR: $70 \pm 10 \%$; Fotofase: 14 horas.

\begin{tabular}{|c|c|c|c|c|c|}
\hline Formulação Comercial & $\mathrm{DC}^{1}$ & Fêmeas/gaiola $(X \pm E P)$ & Ovos/fêmea $^{2}(X \pm E P)$ & $\operatorname{RP}^{3}(\%)$ & Classes $^{4}$ \\
\hline \multicolumn{6}{|c|}{ Experimento I } \\
\hline Testemunha & ----- & $128,90 \pm 12,03$ & $42,46 \pm 5,20 \mathrm{a}$ & ---- & ---- \\
\hline Captan 500 PM & 240 & $105,89 \pm 8,59$ & $38,84 \pm 6,49 \mathrm{a}$ & 8,53 & 1 \\
\hline Delan & 125 & $105,19 \pm 12,29$ & $41,79 \pm 2,31 \mathrm{a}$ & 1,58 & 1 \\
\hline Supracid $400 \mathrm{CE}$ & 100 & $143,35 \pm 3,62$ & $0,00 \pm 0,00 \mathrm{~b}$ & 100,00 & 4 \\
\hline Vertimec $18 \mathrm{CE}$ & 100 & $213,51 \pm 10,24$ & $0,00 \pm 0,00 \mathrm{~b}$ & 100,00 & 4 \\
\hline Dipterex 500 & 300 & $117,06 \pm 15,86$ & $0,00 \pm 0,00 \mathrm{~b}$ & 100,00 & 4 \\
\hline \multicolumn{6}{|c|}{ Experimento II } \\
\hline Testemunha & $\overline{-----}$ & $131,46 \pm 14,35$ & $31,61 \pm 3,68 \mathrm{a}$ & $\overline{-----}$ & ---- \\
\hline Promalin & 9000 & $137,96 \pm 7,89$ & $21,91 \pm 2,27 b$ & 30,69 & 2 \\
\hline Dormex & 1200 & $111,48 \pm 13,33$ & $2,67 \pm 1,03 \mathrm{c}$ & 91,55 & 3 \\
\hline Assist & 4000 & $134,88 \pm 7,56$ & $0,44 \pm 0,25 \mathrm{c}$ & 98,61 & 3 \\
\hline Malathion $1000 \mathrm{CE}$ & 100 & $114,34 \pm 14,07$ & $0,00 \pm 0,00 \mathrm{c}$ & 100,00 & 4 \\
\hline Dipterex 500 & 300 & $98,48 \pm 6,27$ & $0,00 \pm 0,00 \mathrm{c}$ & 100,00 & 4 \\
\hline
\end{tabular}

${ }^{1} \mathrm{DC}=$ Dosagem de Campo (g ou mL produto comercial.100L $\left.\mathrm{L}^{-1}\right)$;

${ }^{2}$ Médias acompanhadas pela mesma letra não diferem significativamente pelo teste de Tukey $(\mathrm{P} \leq 0,05)$;

${ }^{3} \mathrm{RP}=$ Redução na capacidade de parasitismo comparado com a testemunha;

${ }^{4}$ Classes da IOBC para teste de toxicidade inicial sobre adultos: 1=inócuo $(<30 \%), 2=$ levemente nocivo $(30-79 \%), 3=$ moderadamente nocivo $(80-99 \%)$, $4=$ nocivo $(>99 \%)$.

testemunha, o número médio de ovos parasitados por fêmea de $T$. pretiosum foi de 42,46 para o Experimento I e 31,61 para o Experimento II. Este é um importante critério para a validação de testes de seletividade com parasitóide de ovos, uma vez que a metodologia da IOBC/WPRS preconiza um número mínimo de 15 ovos parasitados por fêmea para testes com a espécie T. cacoeciae (Hassan et al., 2000); no entanto, para testes com a espécie $T$. pretiosum, esse limite mínimo ainda não está estabelecido. Rocha \& Carvalho (2004) obtiveram um número médio de 12,90 ovos parasitados por fêmea de T. pretiosum no tratamento-testemunha, valor médio inferior aos verificados neste trabalho. Estudos conduzidos por Maceda et al. (2003) demonstraram que fêmeas de $T$. pretiosum, à temperatura de $25^{\circ} \mathrm{C}$, parasitaram uma média de 5,74 ovos por dia. Esses valores são similares aos resultados obtidos no presente estudo se for considerado que ovos do hospedeiro A. kuehniella foram oferecidos para o parasitismo durante o período aproximado de seis dias ( 144 horas).

As formulações comerciais testadas afetaram distintamente a capacidade de parasitismo de $T$. pretiosum (Tabela 2). $\mathrm{O}$ fungicida Captan 500 PM (captana), na concentração de $0,113 \%$ de ingrediente ativo (i.a.), reduziu a capacidade de parasitismo das fêmeas de $T$. pretiosum em $8,53 \%$ e foi considerado inócuo (classe 1) (Tabela 2). Resultados semelhantes foram encontrados por Hassan (1998b), ao testar Orthocide 83 (captana) na concentração de $0,125 \%$ de i.a. com a espécie T. cacoeciae; no entanto, Captan $83 \mathrm{~W}$ (captana), formulado na concentração de $0,681 \%$ de i.a., foi classificado pelo mesmo autor como levemente nocivo (classe 2). As diferenças obtidas neste caso, provavelmente, se devem à concentração do i.a., que é seis vezes superior à concentração do produto testado neste experimento. Isto evidencia a importância de haver a caracterização dos agrotóxicos utilizados, no advento da publicação de resultados de testes de seletividade. O fungicida Delan (ditianona) foi inócuo (classe 1), reduzindo em apenas $1,58 \%$ a capacidade de parasitismo de fêmeas de T. pretiosum (Tabela 2). Hassan (1998b), ao trabalhar com $T$. cacoeciae, obteve a mesma classificação para o fungicida Delan Flüssing (ditianona).

O inseticida Supracid 400 CE (metidationa) reduziu em 100\% a capacidade de parasitismo de $T$. pretiosum e foi classificado como nocivo (classe 4) (Tabela 2). Para a formulação comercial Ultracid (metidationa), foi também atribuída a classe 4 quando testada para $T$. cacoeciae (Hassan et al., 1998b), evidenciando sua alta toxicidade ao parasitóide. O acaricida-inseticida Vertimec $18 \mathrm{CE}$ (abamectina), na concentração de $0,0020 \%$ de i.a., foi nocivo (classe 4) a T. pretiosum, reduzindo o parasitismo em $100 \%$ (Tabela 2). Resultados similares foram observados por Hassan et al. (1998b) com T. cacoeciae, utilizando o mesmo produto, porém em concentrações superiores. Por outro lado, Rocha \& Carvalho (2004), ao testarem a ação da abamectina em uma concentração de $0,0018 \%$ de i.a. em fêmeas de $T$. pretiosum, verificaram redução na capacidade de parasitismo de $81,40 \%$, ou seja, uma redução de $10 \%$ na concentração do i.a. proporcionou um incremento de quase $20 \%$ na capacidade benéfica do parasitóide. O inseticida Dipterex 500 (triclorfom), utilizado como padrão de toxicidade, foi enquadrado na classe 4 , em ambos os experimentos, com $100 \%$ de redução na capacidade de parasitismo de fêmeas de T. pretiosum (Tabela 2). Este inseticida foi escolhido como testemunha positiva por ter-se revelado nocivo à espécie $T$. cacoeciae na mesma concentração utilizada no presente estudo (Grützmacher et al., 2004).

O regulador de crescimento vegetal Promalin (benziladenina + ácido giberélico) foi classificado como levemente nocivo (classe 2) à espécie $T$. pretiosum, reduzindo em $30,69 \%$ a capacidade de parasitismo das fêmeas (Tabela 2), ficando no limite inferior, quase enquadrado na classe 1. Já o regulador de crescimento vegetal Dormex (cianamida) causou redução de $91,55 \%$ na capacidade de parasitismo das fêmeas e foi considerado moderadamente nocivo (classe 3) a $T$. pretiosum (Tabela 2). A toxicidade destes produtos deve estar relacionada, possivelmente, com a formulação, dosagem utilizada no teste e com efeitos secundários no metabolismo dos insetos, visto que tais produtos não apresentam modo de ação relacionado diretamente à fisiologia dos insetos.

O acaricida-inseticida Assist (óleo mineral), na concentração de $3,024 \%$ de i.a., foi moderadamente nocivo (classe 3), reduzindo em $98,61 \%$ o parasitismo de $T$. pretiosum (Tabela 2). Resultados semelhantes foram verificados por Grützmacher et al. (2004), que realizaram testes para a espécie $T$. cacoeciae com o mesmo produto, porém usando metade da concentração utilizada neste experimento. O inseticida Malathion 1000 CE (malationa), na concentração de $0,100 \%$ de i.a., foi nocivo (classe 4), acarretando $100 \%$ de redução no parasitismo (Tabela 2). Youssef et al. (2004), ao testarem Agrothion (malationa), porém em concentrações superiores, de 0,143\%, observaram uma redução inferior na capacidade de parasitismo de $T$. cacoeciae, que foi de $82,59 \%$. Considerando que foi usado o mesmo ingrediente ativo e concentrações similares, provavelmente, estes resultados diferenciados devam-se a distintos fatores, como componentes utilizados na formulação dos produtos, condições do 
ambiente onde foram conduzidas as pesquisas ou, ainda, diferentes espécies ou "strains" do parasitóide.

Os testes de toxicidade em laboratório sujeitam os insetosteste à máxima exposição aos resíduos das formulações comerciais dos agrotóxicos e constituem a primeira etapa da seqüiência de testes preconizada pelo IOBC/WPRS (Hassan et al., 2000). Conforme a IOBC/ WPRS, os produtos Captan 500 PM e Delan, enquadrados na classe 1 e Promalin na classe 2, não serão mais testados nas etapas subseqüentes. Estes produtos devem ser priorizados na PIM pelos pomicultores no momento da decisão do produto a ser pulverizado na cultura. No entanto, os resultados verificados para os produtos Dormex e Assist, classificados na classe 3, e Supracid 400 CE, Vertimec 18 CE e Malathion 1000 CE, na classe 4, não devem ser extrapolados para condições de campo. É necessário que haja a continuidade dos estudos em casa de vegetação, visando a avaliar a persistência da atividade biológica de cada agrotóxico e, conseqüentemente, a estimativa do risco de impacto a T. pretiosum; e, em última instância, devem ser realizados testes em pomares de macieira que fornecerão resultados definitivos referentes à seletividade desses produtos para T. pretiosum.

\section{CONCLUSÕES}

Em condições laboratoriais, os agrotóxicos testados (\% formulação comercial na calda) Captan 500 PM $(0,240)$ e Delan $(0,125)$ foram inócuos; Promalin $(9,000)$ foi levemente nocivo; Dormex $(1,200)$ e Assist $(4,000)$ foram moderadamente nocivos; Supracid $400 \mathrm{CE}$ $(0,100)$, Vertimec 18 CE $(0,100)$ e Malathion 1000 CE $(0,100)$ foram nocivos a adultos do parasitóide de ovos Trichogramma pretiosum no teste de toxicidade em laboratório. Os agrotóxicos classificados como moderadamente nocivos e nocivos requerem testes subsequientes de persistência biológica do produto em casa de vegetação e de campo.

\section{REFERÊNCIAS}

FERREIRA, A.J.; CARVALHO, G.A.; BOTTON, M.; MENDONÇA, L.A.; CORRÊA, A.R.B. Seletividade de inseticidas usados na cultura da macieira a ovos de Chrysoperla externa (Hagen, 1861) (Neuroptera: Chrysopidae). Ciência Rural, Santa Maria, v.35, p.756-762, 2005.

GRÜTZMACHER, A.D.;ZIMMERMANN, O.; YOUSEF, A.; HASSAN, S.A. The side-effects of pesticides used in integrated production of peaches in Brazil on the egg parasitoid Trichogramma cacoeciae Marchal (Hym., Trichogrammatidae). Journal of Applied Entomology, Berlin, v.128, p.377-383, 2004.

HASSAN, S.A. Guidelines for the evaluation of side effects of plant protection products on Trichogramma cacoeciae Marchal (Hym., Trichogrammatidae). IOBC/WPRS Bulletin, Montfavet, v.21, n.6, p.119-128, 1998a.

HASSAN, S.A. The side effects of 161 pesticides on the egg parasitoid Trichogramma cacoeciae Marchal (Hymenoptera, Trichogrammatidae). Egg Parasitoids, Cali, p.63-76, 1998b.

HASSAN, S.A.; HALSALL, N.; GRAY, A.P.; KUEHNER, C.; MOLL, M.; BAKKER, F.M.; ROEMBKE, J.; YOUSEF, A.; NASR, F.; ABDELGADER, H. A laboratory method to evaluate the side effects of plant protection products on Trichogramma cacoeciae
Marchal (Hym., Trichogrammatidae). In: CANDOLFI, M.P.; BLÜMEL, S.; FORSTER, R.; BAKKER, F.M.; GRIMM, C.; HASSAN, S.A.; HEIMBACH, U.; MEAD-BRIGGS, M.A.; REBER, B.; SCHMUCK R.; VOGT, H. (eds.): Guidelines to evaluate sideeffects of plant protection products to non-target arthropods. Montfavet: IOBC/WPRS, 2000. p.107-119.

IBGE. Levantamento sistemático da produção agrícola: pesquisa mensal de revisão e acompanhamento das safras agrícolas no ano civil. Rio de Janeiro, v.17, n.3, 2005. 74p. Disponível em <http:/ /www.ibge.gov.br>. Acesso em: 25 maio 2005.

KOVALESKI, A.; RIBEIRO, L.G. Manejo de pragas na produção integrada de maçã. Bento Gonçalves: Embrapa, 2002. 8p. (Circular Técnica, 24).

KOVALESKI, A.; SANHUEZA, R.M.V.; RIBEIRO, L.G.; BECHER, W.; BONETI , I.S.; KATSURAYAMA, Y.; PROTAS, J.F. Resultados de pesquisa da produção integrada da maçã (PIM), 2. Bento Gonçalves: Embrapa Uva e Vinho, 2000. p.87-94.

MACEDA, A.; HOHMANN, C.L.; SANTOS, H.R. Temperature effects on Trichogramma pretiosum Riley and Trichogrammatoidea annulata De Santis. Brazilian Archives of Biology and Techonology, Curitiba, v.46, n.1, p.27-32, 2003.

MONTEIRO, L.B. Seletividade de inseticidas a Neoseiulus californicus MacGregor (Acari: Phytoseiidae) em macieira no Rio Grande do Sul. Revista Brasileira de Fruticultura, Jaboticabal, v.23, p.589-592, 2001.

MONTEIRO, L.B.; SOUZA, A.; BELLI, E.L.; SILVA, R.B.Q.;ZUCCHI, R.A. Ocorrência de Trichogramma pretiosum Riley, 1879 (Hymenoptera: Trichogrammatidae) em ovos de Bonagota cranaodes (Meyrick) (Lepidoptera: Tortricidae) em macieira. Revista Brasileira de Fruticultura, Jaboticabal, v.26, p.171-172, 2004.

PARRA, J.R.P. Técnicas de criação de Anagasta kuehniella, hospedeiro alternativo para produção de Trichogramma. In: PARRA, J.R.P.; ZUCCHI, R.A. (Ed). Trichogramma e o controle biológico aplicado. Piracicaba: FEALQ, 1997. cap.4, p.121-150.

PINTO, J.D. Taxonomia de Trichogrammatidae (Hymenoptera) com ênfase nos gêneros que parasitam Lepidoptera. In: PARRA, J.R.P.; ZUCCHI, R.A. (Ed). Trichogramma e o controle biológico aplicado. Piracicaba: FEALQ. 1997, cap.1, p.13-39.

PINTO, J.D.; KOOPMANSCHAP, A.B.; PLATNER, G.R.; STOUTHAMER, R. The North American Trichogramma (Hymenoptera: Thrichogrammatidae) parasitizing certain Tortricidae (Lepidoptera) on apple and pear, with ITS2 DNA characterizations and description of a new species. Biological Control, São Diego, v.23, p.134-142, 2002.

ROCHA, L.C.D.; CARVALHO, G.A. Adaptação da metodologia padrão da IOBC para estudos de seletividade com Trichogramma pretiosum Riley, 1879 (Hymenoptera: Trichogrammatidae) em condições de laboratório. Acta Scientarium, Maringá, v.26, p.315320, 2004.

YOUSSEF, A.I.; NASR, F.N.; STEFANOS, S.S.; ELKHAIR, S.S.A.; SHEHATA, W.A.; AGAMY, E.; HERZ, A.; HASSAN, S.A. The side-effects of plant protection products used in olive cultivation on the hymenopterous egg parasitoid Trichogramma cacoeciae Marchal. Journal of Applied Entomology, Berlin, v.128, p.593-599, 2004. 